\title{
Robustness Analysis of the European Air Traffic Network
}

\author{
Mr. Kuang-Chang Pien \\ Imperial College London, Department of Civil and Environmental Engineering, Centre for Transport \\ Studies, South Kensington Campus, Skempton Building, SW7 2AZ London United Kingdom \\ Email: k.pien11@imperial.ac.uk \\ Phone: +44(0) 2075942705 \\ Corresponding author: Dr. Ke Han \\ Imperial College London, Department of Civil and Environmental Engineering, Centre for Transport \\ Studies, South Kensington Campus, Skempton Building, SW72AZ London United Kingdom \\ Email: k.han@imperial.ac.uk \\ Phone: +44(0) 2075945682

\section{Mr. Wenlong Shang} \\ Imperial College London, Department of Civil and Environmental Engineering, Centre for Transport \\ Studies, South Kensington Campus, Skempton Building, SW72AZ London United Kingdom \\ Email: wenlong.shang12@imperial.ac.uk \\ Phone: $+44(0) 7521549645$

\section{Dr. Arnab Majumdar} \\ Imperial College London, Department of Civil and Environmental Engineering, Centre for Transport \\ Studies, South Kensington Campus, Skempton Building, SW72AZ London United Kingdom \\ Email: a.majumdar@imperial.ac.uk \\ Phone: +44(0) 2075946037

\section{Prof. Washington Ochieng} \\ Imperial College London, Department of Civil and Environmental Engineering, Centre for Transport \\ Studies, South Kensington Campus, Skempton Building, SW72AZ London United Kingdom \\ Email:w.ochieng@imperial.ac.uk \\ Phone: +44 (0)20 75946100
}




\section{Abstract}

The European Air Traffic Network (ATN), comprising of a set of airports and Area Control Centres (ACCs), is highly complex. The current indicator of its performance, air 37 traffic flow management (ATFM) delays, is insufficient for planning and management 38 purposes. Topological analysis of air traffic networks of this kind has highlighted Betweeness Centrality $(B C)$ as an indicator of network robustness, although such an indicator assumes no

40 knowledge of actual traffic flows and the network's operational characteristics. This paper 41 conducts topological and operational analyses of the European ATN in order to derive a more 42 relevant and appropriate indicator of robustness. By applying a flow maximisation model to 43 the network influenced by a range of capacity reductions at the local level, we propose a new 44 index called the Relative Area Index (RAI). The RAI quantifies the importance of an 45 individual node to the performance of the entire network when it suffers from capacity 46 reduction at a local scale. Air traffic data from three typical busy days in Europe are utilised 47 to shown that the $R A I$ is more flexible and capable than $B C$ in capturing the network impact 48 of local capacity degradation. This index can be used to assess network robustness and 49 provide a valuable tool for airspace managers and planners.

\section{Keywords}

51 Air traffic network; robustness; capacity; linear programming 


\section{Introduction}

High air traffic demand in Europe in recent decades has resulted in the severe congestion experienced at both busy airports and en-route airspace. The latter is controlled by Area Control Centres (ACCs). Such congestion has not only caused severe delays and detrimental environmental impacts, but also posed threats to the safety of air travel. Furthermore, with annual air traffic demand in Europe forecast to increase by $2.5 \%$ between 2015 and 2021(EUROCONTROL, 2015), it is expected that the current capacity of the European air transport network will be simply insufficient to cope with this increase. The capacities of ACCs and airports can be defined in terms of maximum number of flights that can be handled in a given period of time.

Compounding this problem of a shortage of capacity on the network is the uneven distribution of air traffic in Europe. According to the Network Operations Report 2013, the top twenty busy airports and congested ACCs were responsible for $67 \%$ of all Air Traffic Flow Management (ATFM) delays in 2013 (EUROCONTROL, 2014).

In order to ameliorate these negative effects and to improve airspace capacity, the Single European Sky (SES) Air Traffic Management (ATM) Research (SESAR) program was launched and one of its features is to change the ATM of Europe from a local to a network level. SESAR envisages that European airspace will be managed as a continuum and as a consequence, network capacity becomes one of the most important Key Performance Areas (KPA). This move to a network level operation and management means that it is essential to understand the fundamental characteristics of the Air Traffic Network (ATN), especially the connections (i.e. the "connectivity") between elements of the network. The mathematical science of topology, which is concerned with network characteristics, provides a viable method for assessing the characteristics of the European ATN. In addition to the topological characteristics, the importance of each constituent node relative to the operation of the entire ATN, in terms of capacity, flow, and bottleneck, needs to be investigated in order to understand their roles and impact in the events of network deterioration or expansion. This paper applies complex network theory, robustness analysis, and network optimization to offer insights on the topological and operational characteristics of the European ATN and provide a quantifiable measure of the importance of its constituent nodes when the network suffers from local distress.

An ATN can be represented by a set of nodes and links. Conventionally, these nodes can be waypoints, en-route airspace or airports, and the links are the flight routes, between these nodes, e.g. airways in en-route airspace. The European ATN is a nonlinear, dynamic and complex system that comprises of numerous heterogeneous components and stakeholders such as airports, Air Traffic Control (ATC) and airspace users. In addition to the heterogeneity of the components, the operational concepts and interactions between different components make ATNs difficult to analyse. Initial studies on the ATNs tend to focus on their topological characteristics, through complex network theory (Holmes, 2004). Comprehensive reviews of existing studies on the application of complex network theory to air transport networks are provided by Sun et al. (2014) and Sun and Wandelt (2014); they found that these studies focus on airports and cities but failed to consider en-route airspace (Lordan et al., 2014; Wei et al., 2014; Zhao et al., 2014). Therefore, Sun et al. (2014) conducted topological analysis on the ATNs in 15 countries, including the USA and major European nations, in which the constituent nodes are both airports and en-route waypoints and the links are flight routes between the nodes. Five topological indices namely: degree, distance strength, Weighted Betweenness Centrality (hereafter referred to as betweenness centrality or $B C$ ), weighted closeness centrality and edge length distribution were calculated. The authors suggest that BC, originally proposed by Freeman (1979), can serve as an index of network robustness and indicates the number of shortest paths passing through a given 
node. A node with high BC is used by more flights and the capacity of it is consequently saturated earlier. Therefore, a network is considered more robust against capacity-reduction at nodes when the network contains nodes with a smaller $B C$ compared to other nodes. However, this conclusion is solely based on topological characteristics and is not validated by using the relevant data of air traffic.

In response to the lack of a suitable robustness index that captures the operational aspect of an ATN, this paper proposes a new index, namely the Relative Area Index (RAI), to assess network robustness based on the actual flight data from three of Europe's busiest days and the published capacity of airports and ACCs. The RAI is developed based on the change in the maximum network flows, calculated through a linear programming (LP) approach, caused by a range of capacity reductions at a given node. Such capacity degradation may be due to local disruptions such as meteorological influence and industrial action. The LP approach is significant in that it provides a theoretical upper bound on the network flow, while taking into account the capacity reduction that occurs at its constituent nodes. Moreover, the estimated maximum flow on each node is significantly correlated with the empirical maximum flow on congested days. Therefore, the LP-based maximum network flows can reasonably reflect the network capacity of the European ATN.

This LP approach, along with the European ATN data that it relies on, are developed using flight profile data provided by the European Organisation for the Safety of Air Navigation (EUROCONTROL). The data were collected on a typical busy day in 2012. This paper extends the analyses of Sun et al. (2014) and Pien et al. (2014) by comparing BC with the new network robustness index (RAI) for the European ATN. The latter index takes into account a range of capacity reduction and its impact on the network operation, rather than simply removing the node, and thus is more realistic in characterizing the robustness of the ATN.

This paper not only calculates the topological index (betweenness) of the entire European ATN for the first time in the literature, but also provides a validated tool (RAI) for Europe's airspace managers and planners to assess network robustness in the event of any local deterioration of nodal capacity. The RAI is compared with the actual traffic demand and published capacity at each node and shown to have the potential to identify the important nodes in the network.

The rest of this paper is organised as follows. Section 2 introduces an overview of robustness analysis in transport networks and relevant literature. The development of the RAI is also introduced. The European ATN flight profiles, capacity constraints, and network structures are described in detail in Section 3. Section 4 presents the linear programming approach for traffic flow maximisation. Section 5 presents the results and analysis on the European ATN. The findings are discussed in Section 6 prior to the conclusions in Section 7.

\section{Robustness Analysis}

Since the definition of robustness varies in different fields, it is pertinent to review the definitions and their context in the literature to define the robustness of an ATN. Furthermore, the current Key Performance Indicator (KPI) of network capacity in the European ATN is introduced. Based on previous research, the conventional index of BC is described. Finally, we develop a new index, the RAI, to assess the robustness of the European ATN.

\subsection{Literature review}

The robustness of transport networks has been a central focus of network planning and management. It is often investigated in different performance areas such as stability, 
resilience and permanence to assess the capability of handling worsened or perturbed conditions of the network.

Since there are numerous categories of networks, there is no universal definition of network robustness, though the following three are highly relevant for this paper:

- "The degree to which a system or component can function correctly in the presence of invalid inputs or stressful environmental conditions" (Geraci et al., 1991).

- 'The degree to which a system is capable of functioning according to its design specifications in the case of serious disruptions" '(Immers et al., 2004).

- The robustness of an electrical network is defined as the capability of maintaining its structure and function when the network is exposed to perturbations (Holmgren, 2007)

Given these definitions, the robustness of a given system or component is therefore the capability of maintaining its function or performance in order to cope with disruptions, perturbations and stressful conditions. Whilst useful, these studies focus on either an individual system or a component. In order to cope with the robustness at network level, it is illustrative to consider the experience of research on transport networks as outlined below.

- Sakakibara et al. (2004) proposed a topological index to evaluate the depressiveness and concentration of road networks in the presence of disasters. They suggested that a network is considered robust when it is able to minimize the isolation of districts when catastrophic disasters occur.

- Scott et al. (2005) proposed the Network Robust Index (NRI) to identify the critical links of a highway network. This index was calculated by comparing the changes in travel time cost of the network when a given highway segment (i.e., network link) is removed from it. Compared to the conventional method of using the ratio of volume to capacity which can only reflect the congestion at local level, the NRI provides better planning solutions to enable the identification of critical links at the network level.

- Nagurney and Qiang (2007a) proposed a network efficiency measure to assess the efficiency of congested networks. Their approach is used to rank the importance of a given link by comparing the change of total travelling costs when the link is removed from the network. In their later work Nagurney and Qiang (2007b) use the relative change of network efficiency as an index to assess network performance when the capacities of all links are reduced by the same percentage. The authors therefore developed the Relative Total Cost Index (RTCI) to assess the robustness of networks against a global decrease of link capacities (Nagurney and Qiang, 2009). Compared to removing links from the network, this approach provides a more realistic method of assessing network robustness when disturbance occurs on any of its constituent components.

Based on these studies, the robustness of a network can be defined as the capability of maintaining network performance while its functioning components, namely nodes and links, are under stress. With this definition in mind, we conduct robustness analysis by taking into account the topological and operational characteristics of the European ATN, and treating network capacity as the main key performance area (KPA). In particular, we consider the maximum network flow, which is obtained through an optimization procedure, as the key indicator of network capacity. Accordingly, the robustness of the European ATN in this paper is related to the capability of delivering the maximum traffic flows against degradation of nodal capacities. In the following sections, the current KPIs of network capacity and the conventional index of network robustness are reviewed.

\subsection{KPI of network capacity: ATFM delays}

Currently, ATFM delays are used as the KPI to monitor network capacity (EUROCONTROL, 2007). This is the duration between the last take-off times requested by the aircraft operator and the take-off slots allocated by the central flow management unit 
namely the Network Manager Operations Centre. This duration follows an air traffic flow regulation, which is subsequently communicated by the flow management positions to an airport or en-route centre.

However, there is a major deficiency with this measure since ATFM delay is not a direct measure of capacity but rather is a proxy that reflects the extra time caused by capacity shortages, which are in turn caused by various factors at airports and in en-route airspace. Since it is an indirect measure, there is an inherent inaccuracy in identifying the important nodes in a network. For instance, Maastricht ACC highlights the limitation of ATFM delays as an indicator. Although the air traffic demand in Maastricht ACC is amongst the highest in Europe, the ATFM delays are relatively low. However, since the nodes with high ATFM delays are considered as bottlenecks in the European ATN, we use them to conduct an intuitive verification of the other indices.

\subsection{Topological Index: Betweenness Centrality (BC)}

In complex network theory, nodes within a network may be ranked by using different centrality measures (Wasserman, 1994). The rank of a given node reflects the measurement of some particular structural property in the network. Several centrality measures, such as degree, betweenness, and closeness, can be used to rank the importance of nodes, among which betweenness is the most widely used as an index of network resilience and robustness (Holme et al., 2002; Newman, 2001). The betweenness centrality (BC) of a node is defined as the probability that it lies in the shortest path(s) between all origin-destination (OD) pairs (Dehmer, 2011; Di Paolo et al., 2011). Mathematically, the conventional formulation of $B C$ can be presented as:

$$
B C_{i}=\sum_{m, n \in \boldsymbol{v}} \frac{S_{m n}^{i}}{S_{m n}}
$$

where $B C_{i}$ is the betweenness of the node i. $S_{m n}$ is the total number of shortest paths between any pair of nodes $(m, n)$ and $S_{m n}^{i}$ is the number of shortest paths passing through the node $i$.

Newman (2001) states that the higher the $B C$, the more influential is the node. The largest increase in the travel distance among nodes occurs when the node with the highest $B C$ is removed. This explains intuitively the importance of high- $B C$ nodes relevant to the overall performance of the network. In addition, a network with many low- $B C$ nodes is more robust than that with many high- $B C$ nodes. Brandes (2001) notes that $B C$ is the most frequently employed centrality index in the analysis of social networks; and it is mostly based on shortest paths. Barrat et al. (2004) claim that nodes in the inner network are more likely to be used by shortest paths than those in the outer network. Therefore, in an ATN, it can be intuitively assumed that the airspace nodes are more likely to be passed by the shortest paths and the nodes with high $B C$ are more likely to handle more traffic, when the traffic demands are uniformly distributed. Travellers within a network tend to choose the shortest paths and as a result, the nodes with high $B C$ tend to be used by more travellers.

However, in the 'real world' of transport operations, nodes with high $B C$ are not necessarily busy (or heavily loaded) due to the fact that the traffic network flows are jointly determined by a number of factors such as travel demand distribution, complex decision factors, and route choice patterns that are not based on shortest paths (i.e., not all-or-nothing assignment). For example, Cats and Jenelius (2014) developed a dynamic-stochastic model to evaluate the impacts of disruptions, and demonstrated that $B C$ may not be a good indicator of link importance in a road network. Guimera and Amaral (2004) modelled a world-wide airport network and showed that the airports with high $B C$ are not necessarily hubs. They 
argued that geo-political constraints play an important role in the growth of airport networks and other critical infrastructure. In addition, although both are modelled as nodes in an ATN, the roles of airports and ACCs are different. An airport not only acts as an origin/destination but also as a transfer node (hub), which means that it serves as the en-route node of a complete trip from the origin to the destination. However, the conventional formulation shown in (1) treats the airports and ACCs equally without considering their heterogeneity. Therefore, this conventional $\mathrm{BC}$ needs to be tailored and improved to accommodate the unique characteristics of the ATN. All these aforementioned factors contribute to the consensus that $\mathrm{BC}$ may no longer be sufficient to assess the robustness of a complex network such as the ATN. This paper contributes to this line of research by proposing the relative area index (RAI), which serves as an alternative robustness index that captures the ATN's flow capacity and certain aspects of its operational features.

\subsection{Relative Area Index (RAI)}

Built upon the definition of robustness, the $R A I$ assesses and quantifies the impact on the maximum network flow of a wide range of capacity reductions that occur at a local (nodal) level. Its derivation is outlined below.

The potential reduction of nodal capacity in the ATN is parameterised with $u$, which is termed the degradation parameter $(D P)$. We employ the notation $F_{\max }^{i}(u)$ to represent the maximum network flow when a capacity reduction expressed by $u$ is applied to node $i$. The procedure of finding the maximum network flow with a given degradation parameter amounts to a linear program, as we describe in detail later in Section 4.

In this study, two types of DPs are considered: (1) percentage based DP; and (2) absolute value based $D P s$. The Percentage- $D P\left(u^{P}\right)$ ranges from $0 \%$ to $100 \%$, and represents the reduction of the subject node's capacity in percentage. The Absolute- $D P\left(u^{A}\right)$ ranges from 0 to 50 (in flights per hour). The value 50 is chosen since it is about half of the capacity of the node with the smallest capacity (LA PALMA airport). Anything significantly larger than 50 may cause the reduced capacity at some nodes to be negative, which is clearly infeasible. In order to distinguish these two methods of capacity reduction, we denote the $R A I$ based on Percentage- $D P S$ by $R A I_{P}$, and the RAI based on Absolute-DPs by $R A I_{A}$.

Since the network maximum flow problem is formulated as an optimization problem constrained by nodal capacities, we deduce that $F_{\text {max }}^{i}(u)$ is a monotonically decreasing function of $u$, where a larger $u$ represents greater capacity degradation at the relevant node. Error! Reference source not found. illustrates the rationale behind RAI. As shown in Error! Reference source not found.(a), two functions, $F_{\max }^{i}(u)$ and $F_{\max }^{j}(u)$ corresponding to nodes $i$ and $j$ respectively, indicate that node $i$ is the more important one as far as maximum network flow is concerned. This is because the same level of degradation yields a smaller network flow when applied to node $i$ than node $j$. In general, the lower the function $F_{\text {max }}^{i}(u)$, the more detrimental it is to reduce the capacity at node $i$. In order to further quantify such an observation, we consider the area formed by the graph of $F_{\max }^{i}(u)$, the vertical line passing through $u_{T}$, and the horizontal line passing through $F_{\max }^{i}\left(u_{0}\right)$. Such an area is illustrated as the shaded part in Error! Reference source not found.(b). It is understood that the large the area, the more critical is the node. For an ATN, the more critical nodes there are, the less robust is the network against capacity reductions. Finally, we note that two distinct functions may yield the same area, as shown in Error! Reference source not found.(c). Thus, in order to distinguish such circumstances, we introduce the weighting parameters (WP) $w(u)$. The WPs assigns different priorities to different range of capacity reductions, and may depend on the node of interest, type and nature of capacity reduction, 
299

and application scenarios. For example, if the main cause of capacity reduction is scheduled maintenance, which has a mild effect on airport capacity, then lower values of $u$ will be assigned higher weights. Therefore, in Error! Reference source not found.(c) the node $i$ (solid line) corresponds to larger weighted area than node $j$ (dashed line), and thus is more critical.

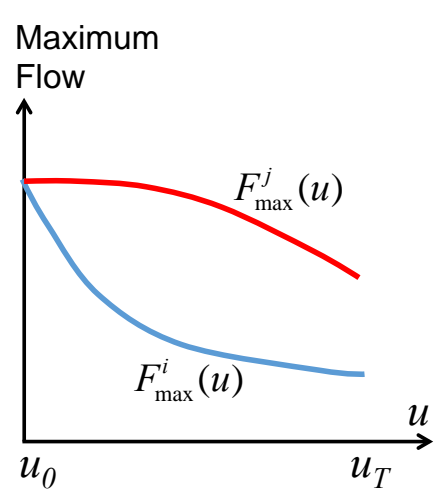

(a)

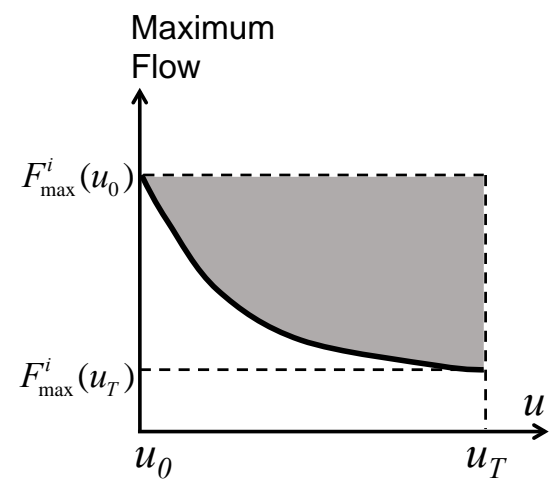

(b)

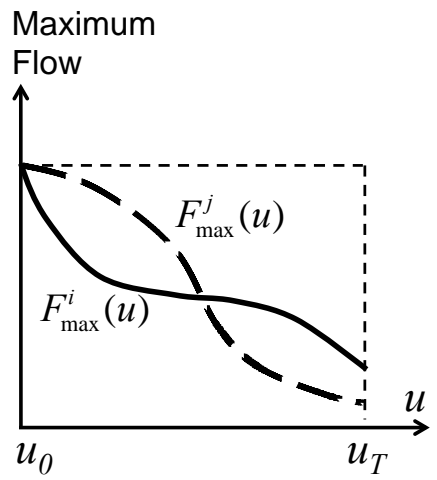

(c)

Figure 1. Illustration of the relative area index.

In this study, we select three types of WPs. The first type assumes equal weights for all values of the degradation parameter $u \in\left[u_{0}, u_{T}\right]$. The second type of WP assigns higher weights to small values of $u$, in contrast to the third type, which assigns lower weights to the small values. By using these three sets of WPs, it is possible to gain insights into the (global) influence of the local capacity-reductions. With this in mind, we formulate the relative area index (RAI) for a given node $i$ as:

$$
R A I^{i}=\frac{\int_{u_{0}}^{u_{T}} w(u)\left(F_{\max }^{i}\left(u_{0}\right)-F_{\max }^{i}(u)\right) d u}{\int_{u_{0}}^{u_{T}} w(u) F_{\max }^{i}\left(u_{0}\right) d u}
$$

We note that a normalisation factor (denominator) is applied to the aforementioned weighted area (numerator). As we previously mentioned, percentage-based and absolute-value-based degradation parameters $\left(u^{P}\right.$ and $\left.u^{A}\right)$ are considered; namely, $u^{P} \in[0,1]$ and $u^{A} \in[0,50]$.

In general, the $R A I$ defined for a given node encapsulates the global impacts of capacity reductions at this node, which depend not only on the network topology, but also on the configuration of OD pairs, flight routes, and nodal capacities. The RAI is defined in terms of a flow maximisation problem, and is not available in a closed form. Thus, it is difficult to predict the distribution of RAIs using simple topological indicators such as $B C$. There are, however, a few simple interpretations of the $R A I$. In particular, it is reasonable to expect that nodes with higher capacities should in general have larger (percentage-based) $R A I_{P}$ values than those with lower capacities based on the following observation: the percentage-based capacity reduction at a high-capacity node results in greater absolute reductions. However, as we subsequently show in Section 5.2, some nodes in the European ATN possess $R A I_{P}$ that are quite counter-intuitive, as suggested by their size, capacity, and significance to the network. 


\section{The European Air Traffic Network}

The key elements required to conduct the robustness analysis are the weighted adjacency matrix and the maximum network flow estimation method. The former is used to calculate betweenness, while the latter is used to calculate the RAIs for the network against the capacity reductions at every node. This section introduces the European ATN and the required data for calculating $\mathrm{BC}$ and the maximum network flows.

\subsection{Data processing}

According to the latest European Network Operations Plan (EUROCONTROL, 2014), the European ATN comprises of 41 countries, and the en-route airspace of Europe is controlled by 64 ACCs. In order to monitor the air traffic at the network level, EUROCONTROL records detailed daily profiles of all flights in Europe at the levels of the ACCs, en-route sectors and waypoints - the latter of which are often associated with navigation aids, in particular radars. Each flight profile represents a flight route that uses a sequence of nodes. This dataset provides the information on the times and coordinates of each flight at every node. In order to investigate the robustness at the network level, airports and ACCs are employed as the constituent nodes of the European ATN.

Flight profiles recorded on $1^{\text {st }}$ July 2012 were used to extract the required information. On this day, a total of 28,904 flights were scheduled, among which 28,885 flight profiles were recorded by the radars. In total, 28,753 flight profiles are used for this study, with the remainder excluded since they either used unrecognized airports or passed through unrecognized airspace.

The average ATFM delays per flight on this particular day were among the highest in 2012. This date also falls within the European summer, which is the season with the highest traffic demand throughout a year. The advantage of using the flight profiles for this day is that the high ATFM delays enable us to capture the spatial configuration of traffic congestion, while the high traffic demands (flight routes) provide sufficient information on the connectivity between nodes. The ATFM delay data are provided by EUROCONTROL.

\subsection{Network topology}

The European ATN can be represented as a directed graph, in which the nodes represent airports and ACCs. A critical notion is connectivity, which can be defined as a binary state that exists between any two nodes in the network, taking a value one if the two nodes are connected by a link and zero otherwise. Unlike many traditional transport networks, the capacity constraints in an ATN are imposed at the nodes (airports and ACCs) rather than on the links. The declared capacities at airports and en-route airspace are applied to prevent the relevant node from overload through the mechanism of air traffic flow management (ATFM), which includes re-routing and the imposition of flow regulation. Air traffic at European airports and in en-route airspace is required to comply with the declared capacities. Based on these characteristics, the European ATN can be regarded as a capacitated transport network and, consequently the traffic flows within it cannot exceed the theoretical maximum. The fundamental components of a capacitated transport network are the constituent nodes and links, as detailed below. These components can be updated and reselected by using different techniques with the latest operational reports when they are available.

\subsubsection{Constituent nodes}

Based on the flight profiles, this network consists of 850 nodes, which include 784 airports, 64 ACCs and two external nodes. The external nodes are used to represent airports and airspaces that are external to the European region. According to Pien et al. (2014), the 
airports can be categorized as 'busy airports' and 'less busy airports'. A total of 67 busy airports were selected based on the top airports listed in EUROCONTROL (2013c) and EUROCONTROL/FAA (2009). These busy airports carry about $60 \%$ of the overall flights in Europe, while the less busy airports carry the remaining $40 \%$.

The capacity data at less busy airports are not publically available. In order to overcome this difficulty, we treat the less busy airports collectively as one or several aggregate nodes. Figure 2 shows the method of creating an aggregate node that represents a group of less busy airports that are of interest to a particular ACC. The connectivity among these less busy airports and relevant ACC is identified through the flight profile data. It is worth noting that some less busy airports may be adjacent to more than one ACC. In this case, we assign this airport to the ACC that contains the most number of flights originating from it. Following this rule, we are able to assign each less busy airport to a unique ACC; and less busy airports assigned to the same ACC are aggregated to form an aggregate node. By using the aggregate nodes, the issue of unknown capacities of the less busy airports is circumvented since active bottlenecks can occur only at the level of ACCs that watch over the less busy airports, rather than at these airports themselves; in other words, the capacities of individual less busy airports are not explicitly needed for the flow maximization problem. Applying the aggregate nodes also reduces the network size while maintaining the connectivity between the less busy airports and their adjacent ACCs. The reduced network contains 197 nodes, including 67 busy airports, 64 aggregate airports, 64 ACCs and 2 external nodes.

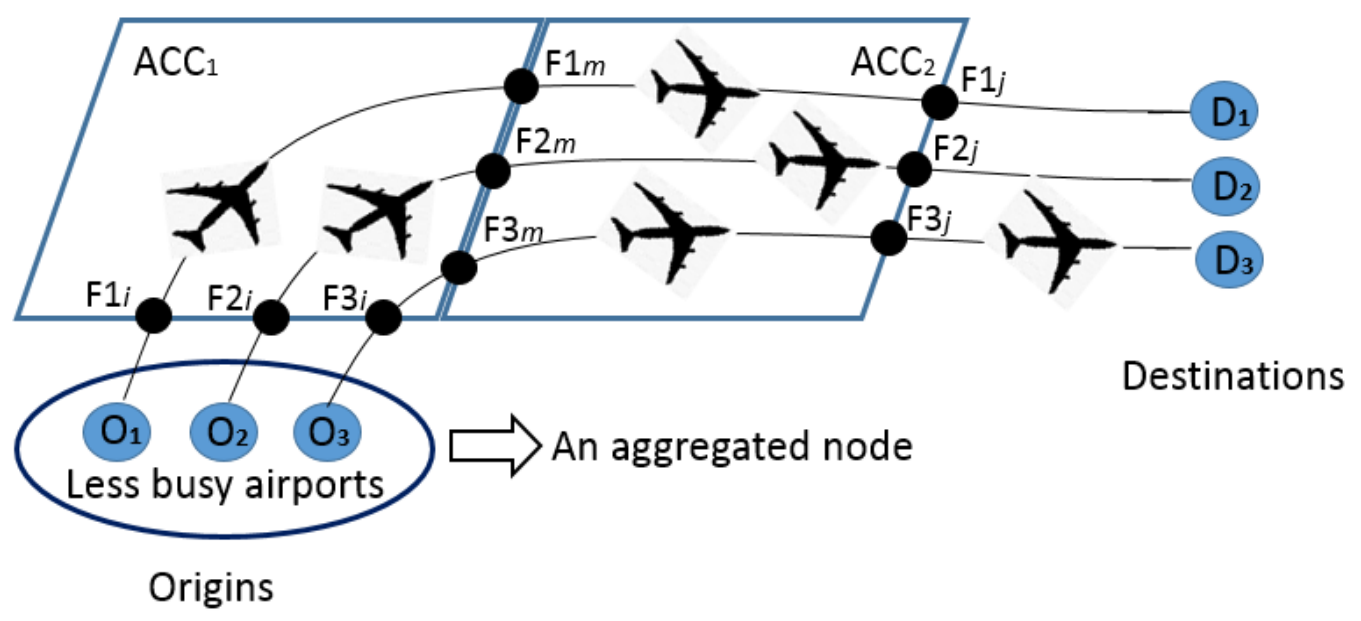

Figure 2. Illustration of the aggregate airports.

The flow maximization problem we shall consider later employs a static flow modelling approach; that is, we consider the stationary flows in the network on a daily basis, without explicitly considering the within-day dynamics of various variables. Thus, the capacities of the airports and ACCs (in flights per day) as we consider in this paper are calculated from the declared capacity (in flights per hour) by a multiplication factor of 16 and 24 , respectively, meaning that the operational hours at the airports and ACCs are 16 hours and 24 hours per day. Although the air traffic demand and traffic intensity are unevenly distributed over the duration of operational hours, the static modelling approach for ACCs and airports enables the calculation of the theoretical maximum network flows, which can serve as a theoretical upper bound of network capacity.

\subsubsection{Constituent links}

A directional link in the network is defined as the connectivity between an ordered pair of nodes. The link between any pair of nodes is established if the flight profile data 
suggests the consecutive passing of the two nodes by at least one flight. The weight of the link is defined to be the average flying distance from its tail node to its head node, which is obtained using the actual flight data.

Issues arise from the different spatial characteristics of airports and ACCs. In particular, an airport can be regarded as a single point with negligible size while an ACC usually covers a significantly larger area. The distance between an airport (or an aggregated airport) to its adjacent ACC can be calculated by averaging the distance between the airport and the entry point to its adjacent ACC, and such entry points are recorded in the dataset of flight profiles.

However, the flying distance between two ACCs cannot be directly calculated by using the distance between their centres, due to their relatively large areas and irregular shapes (ACC boundaries in Europe follow national borders). Assuming that $N$ flights are flying through node $i$ and $j$, the flying distances of these $N$ flights between the node $i$ and $j$ are $D_{(i, j)}^{N}$. The weight of the link between $\operatorname{ACC}_{i}$ and its adjacent $\operatorname{ACC}_{j}\left(L_{(i, j)}\right)$ can be formulated as:

$$
L_{(\mathrm{i}, \mathrm{j})}=\frac{1}{2 \mathrm{~N}} \sum_{N=1}^{N} D_{(i, j)}^{N}
$$

\subsubsection{Validity of the network data}

As mentioned earlier, the network topology and the flow maximization problem are based on the flight data on $1^{\text {st }}$ July 2012. Datasets collected on two additional busy days, namely $28^{\text {th }}$ and $29^{\text {th }}$ July 2012, are used to validate the network data. More specifically, we use correlation coefficients to assess the similarity of the network adjacency matrices calculated from the data on these three days. On the other hand, the Mean Absolute Percentage Error (MAPE) and correlation coefficients are used to compare the maximum flows (details to follow in Section 4) calculated by using data on these three days.

The correlation coefficients for the network adjacency matrices on the three days are above 0.85 . In addition, the correlation coefficients among the maximum flows on these three days are above 0.99 and the MAPEs are less than $0.5 \%$. This shows consistency of our dataprocessing method and the validity of the resulting network topological information and maximum flow data.

\section{Traffic Flow Maximization}

The problem of finding the theoretical maximum network flow subject to the network topology and capacity constraints is formulated as a linear program. In this section, we first recap the LP approach for maximising network flows originally proposed by Pien et al. (2014). This is followed by an interpretation of the $R A I$ in relation to the Lagrange Multipliers (LM), which is relevant to the linear program and the marginal costs of local capacity reduction.

\subsection{Network flow maximisation formulated as a linear program}

As mentioned earlier, the European ATN is considered as a capacitated transport network in which the operations at airports and in ACCs are subject to their individual capacity limits. The maximum network flows are estimated by using a LP approach. The estimated maximum flow on each node is significantly correlated to the empirical maximum flow on congested days. Therefore, the estimated maximum network flows can reasonably reflect the network capacity of the European ATN. 
Figure 3 depicts the structure of the network and the flight paths therein. The network comprises of busy airports, aggregated airports and ACCs. An aircraft departs from its origin airport and flies along its flight path in en-route airspace to its destination airport. The flights that use European airspace can be categorised into three groups: intra-Europe flights, intercontinental flights, and over-flights. The flights flying along the flight paths $p_{1}$ and $p_{2}$ are intra-Europe flights that fly between two European airports. The flights along paths $p_{3}$ and $p_{4}$ are inter-continental flights that fly from an European airport to an airport outside Europe or vice versa. The group of over-flights $p_{5}$ represents the flights passing European airspace without using any European airports.

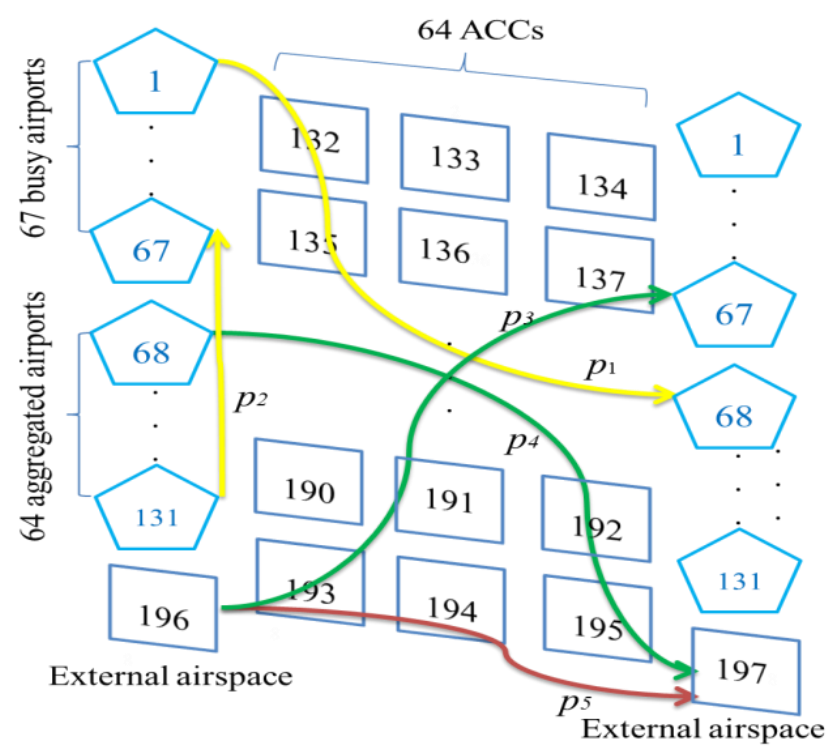

Figure 3. Structure of the European ATN.

We consider a network with given sets of paths $(P)$ and origin-destination (OD) pairs $(W)$. For any $(i, j) \in W$ where $i$ denotes origin and $j$ denotes destination, let $P_{i j}$ be the set of flight paths connecting $i$ to $j$. Each flight path is represented as a set of nodes (airports and ACCs) it traverses. The relationship between paths and nodes is encapsulated by the pathnode incidence matrix $\left(\delta_{p v}\right)$ :

$$
\delta_{p v}=\left\{\begin{array}{lr}
1 & \text { if } v \in p \\
0 & \text { otherwise }
\end{array}\right.
$$

where $v$ denotes a node, and $p$ denotes a path. In addition, each node $v$ in the network has a flow capacity $C_{v}$.

The maximum network flow problem is formulated as follows. The objective is to maximize the path-based flows in the entire network:

$$
\max \sum_{p \in P} f_{\boldsymbol{p}}
$$

475 where $f_{p}$ denotes the flow along path $p$. The constraints include:

476 Flow capacity constraint: $\forall k \in \boldsymbol{A N}$ (the set of nodes)

$$
\sum_{p \in P} \delta_{p k} f_{p} \leq C_{k}
$$


Nonnegativity: $\forall p \in P$ (the set of flight paths)

$$
f_{p} \geq 0
$$

477 The influence of capacity reduction at a given node on the maximum network flows can be 478 obtained by solving a family of such linear programs, each with a decreased flow capacity at 479 a given node. This procedure is the basis for calculating the RAI.

480

481

482

483

484

485

486

487

488

489

490

491

492

493

494

495

496

497

498

499

500

501

502

503

504

505

506

\subsection{Lagrange multiplier and $R A I$}

The use of the Lagrange multiplier is a common approach to solving optimization problems (Jahn, 2007). The Lagrange multiplier is the rate of change of a quantity being optimized as a function of the constraint variable. In the case of maximizing network flows, the Lagrange multiplier is the sensitivity of the maximum network flow with respect to the change in the capacities. It can be interpreted simply as the marginal cost (gain) of the network maximum flow with respect to an infinitesimal reduction (increase) in the nodal capacity. Lagrange multipliers are zero at non-bottleneck nodes, which corresponds to the complementarity conditions arising from duality; this means that small changes in capacity at these non-bottleneck nodes have no effect on the maximum network flows. The higher the Lagrange multiplier, the more critical the node is to the overall throughput of the network.

The Lagrange multiplier is related to the $R A I$, since the former is precisely the derivative of the function $F_{\text {max }}^{i}(u)$ evaluated at 0 , with a negative sign. However, the $R A I$ presents knowledge of the rest of the function for $u \in(0 \%, 100 \%]$ or $(0,50]$ whereas a Lagrange multiplier only shows the initial trend of the curve when the capacity reduction is small; see Figure 4 for an example. In Figure $4(a)$, the initial decrease of the curve $F_{\max }^{i}(u)$ is small, indicating a small Lagrange multiplier. However, as the capacity reduces further, the maximum network flow drops drastically. In comparison, Figure 4(b) shows a curve with steeper initial decrease, but which then stabilises for larger $u$. From this figure, we see that the Lagrange multiplier and the RAI may provide very different information regarding the importance of the subject node, despite their relationship illustrated above.

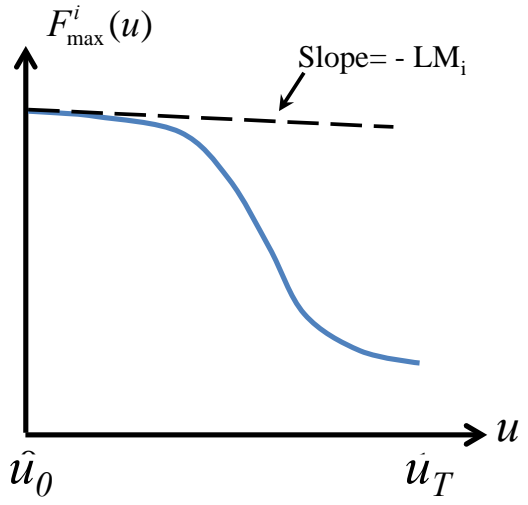

(a)

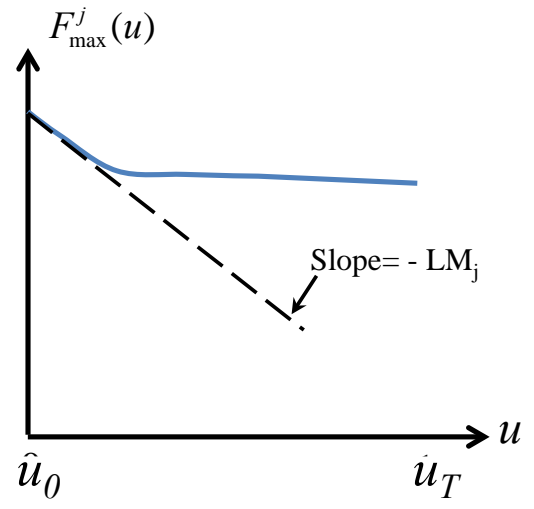

(b)

Figure 4. Relationship between the Lagrange multiplier and the RAI

We see from these simple examples that the $R A I$ is a more comprehensive performance indicator for a node subject to capacity reductions than the Lagrange multiplier, as the former captures a whole range of capacity reductions. Moreover, the use of 
appropriately defined weighting parameters, shown in Eq. (2), makes the $R A I$ quite flexible in addressing a target range of capacity reductions, which can be user defined.

\section{Results and Analysis}

In this section, we present results related to the $B C$ and $R A I$, and provide some discussions on their managerial insights in the context of air traffic management on a network level. Since the focus and the underlying assumptions of $B C$ and $R A I$ are different, we compare $B C$ to traffic load and $R A I$ to nodal capacity. The rationale behind these comparisons is that nodes with higher $\mathrm{BC}$ tend to carry more traffic load since most flight routes follow the shortest paths; on the other hand, nodes with higher capacity are intuitively more important to the maximum flow of the entire network. In order to simplify our analysis and to distinguish between airports and airspaces, we extract the top ten airports and airspaces in each category $(B C \& R A I)$ and highlighting nodes that are significant according to both the indicators. Since the aggregated nodes are used to maintain the network structure and do not represent solid locations, we exclude them from the ranking list. The rankings of the airports and airspaces are then compared with the empirical data on air traffic load, nodal capacities, and ATFM delays.

In subsequent presentation, unless otherwise specified, we assign ID numbers $1-67$ to the 67 airport nodes, 68 - 131 to the 64 aggregate airport nodes, and 132 - 195 to the 64 ACC nodes.

\subsection{Betweenness centrality $(\mathrm{BC})$}

Figure 5 displays information of the $B C$ in the entire network. Compared to the airport nodes (ID 1 - 131), the ACC nodes (ID 132 - 195) overall have larger BCs. An intuitive explanation is that the ACC nodes in the network can be considered as inner nodes while the airport nodes can be regarded as outer nodes (see Figure 3 for an illustration). Thus the inner nodes tend to have higher BCs, an observation consistent with the work of Barrat et al. (2004).
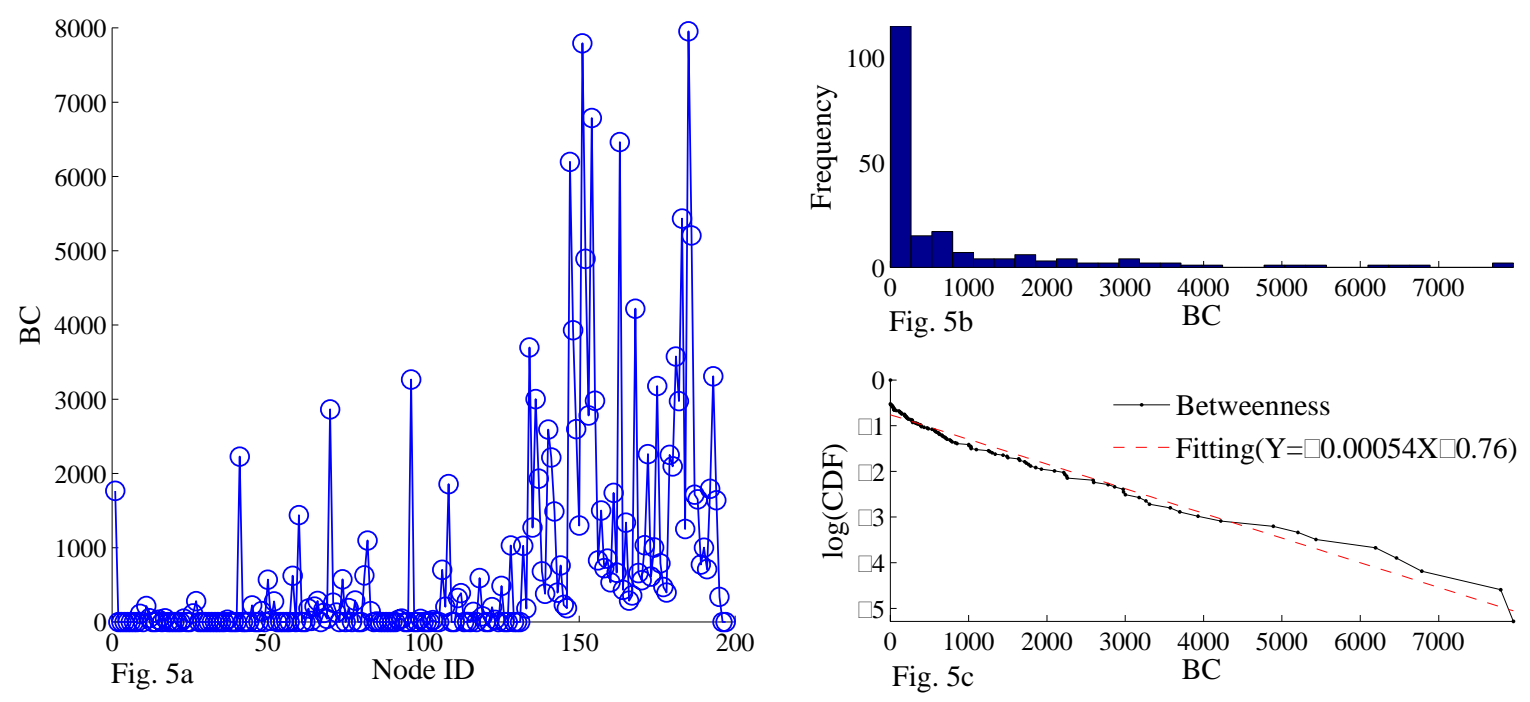

Figure 5. $\mathrm{BC}$ in the European air traffic network.

Figure $5 \mathrm{~b}$ and Figure $5 \mathrm{c}$ show the histogram and the cumulative distribution function (CDF) of $B C s$, respectively. The $\mathrm{CDF}$ of the weighted $B C$ can be fitted by an exponential function: $P(\geq b) \sim e^{-0.00054 b}$. Such a fitting is a common approach in network science to quantify the robustness of a network based on topological indices such as the BC; the reader 
is referred to Sun et al. (2014) for a more elaborated discussion and more examples of such fitting for a variety of other air traffic networks.

The ten airports and airspace with the highest air traffic loads and BCs are listed in Table 1. It is notable that airport nodes with high $B C s$ do not necessarily have the highest traffic loads, and vice versa. The airports handling high traffic demands all locate on the capitals or economic centres rather than the high- $B C$ airports. Therefore, the $B C$ is not capable to capture the high-traffic airports and this results is consistent with Cats and Jenelius (2014) and Guimera and Amaral (2004).

\begin{tabular}{|c|c|c|c|c|}
\hline \multirow[b]{2}{*}{ Rank } & \multicolumn{2}{|c|}{ Airport } & \multicolumn{2}{|c|}{ Airspace (ACC) } \\
\hline & $B C$ & Traffic load & $B C$ & Traffic load \\
\hline 1 & VALENCIA & FRANKFURT & GENEVA & LONDON \\
\hline 2 & BRUSSELS & PARIS CDG & BREMEN & MAASTRICHT \\
\hline 3 & GENEVE & LONDON HEATHROW & MUNICH & KARLSRUHE \\
\hline 4 & WIEN SCHWECHAT & SCHIPHOL AMSTERDAM & ROME & MUNICH \\
\hline 5 & MAKEDONIA & MADRID BARAJAS & MARSEILLE & MARSEILLE \\
\hline 6 & ISTANBUL SABIHA & MUENCHEN & MALMO & ROME \\
\hline 7 & TRONDHEIM VAEMES & ISTANBUL ATATURK & ZURICH & LONDON TC \\
\hline 8 & CATANIA FONTANAROSSA & ROME/FIUMICINO & LANGEN & $\underline{\text { PARIS }}$ \\
\hline 9 & NICE & BARCELONA & AMSTERDAM & LANGEN \\
\hline 10 & BIRMINGHAM & PALMA-DE-MALLORCA & PARIS & BREST \\
\hline
\end{tabular}

Table 1. Top ten airports and airspaces that have the highest $B C s$ and traffic loads.

In terms of the $B C s$ of airspace (ACC) nodes, five high- $B C$ ACCs, namely Munich, Rome, Marsellie, Langen and Paris, also handle high air traffic. This result indicates that the $B C$ is relatively more capable of capturing the important nodes with high traffic demands in airspace than at airports. However, the corresponding $B C$-rankings of the top three hightraffic ACCs, namely London, Maastricht and Karlsruhe are extremely low. Therefore, $B C s$ cannot fully capture the traffic demands in the real world of operational traffic.

\subsection{Relative area index $(R A I)$}

According to our discussion of the $R A I$, the capacity of a node may be reduced by a certain percentage or by an absolute value. The resulting $R A I_{P}$ and $R A I_{A}$, respectively, are presented and analysed in this section to examine the influence of capacity reductions on the network capacity. We use Spearman's rank correlation coefficient $(r)$ and $p$-value $(p)$ to measure the statistical dependence and the statistical significance between different sets of results. The detailed results of the RAIs and the $B C s$, as well as the empirical data, of a selection of nodes are presented in the Appendix.

\subsubsection{Relative area index with capacity reduction by percentage $\left(R A I_{P}\right)$}

In this section, we illustrate three sets of $R A I_{P}$ over the entire European ATN in Figure 6. These three sets of $R A I_{P}$ are calculated by using three different weighting parameters that emphasize the capacity reduction at different levels. The first weighting parameter treats the importance of capacity reductions at all levels equally, and is indicated as 'Constant' in Figure 6. The second and third weighting parameters assign a low (high) weight to low capacity reduction and a high (low) weight to high capacity reduction; in particular, they vary the weight from 0 to 10 and 10 to 0 , respectively. Therefore, the second and third weight parameters emphasize the importance of higher and lower capacity reductions at each node, respectively. 


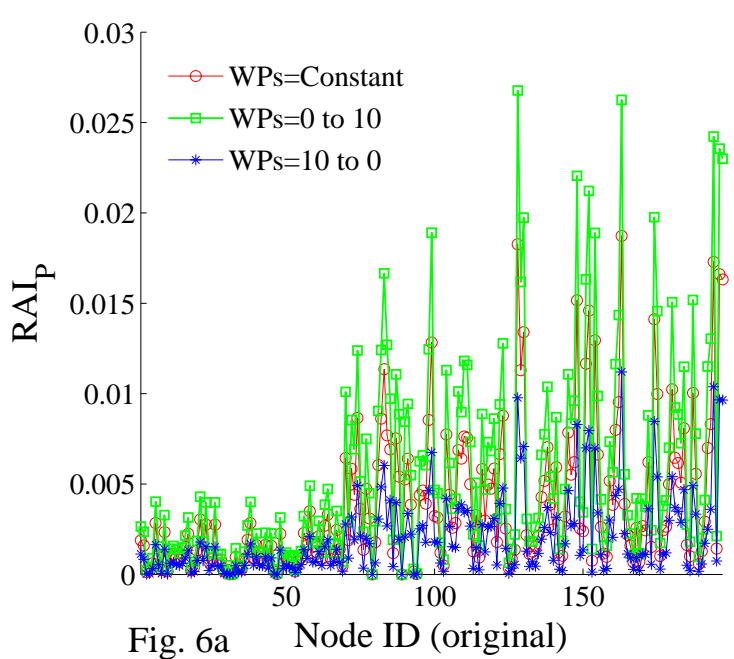

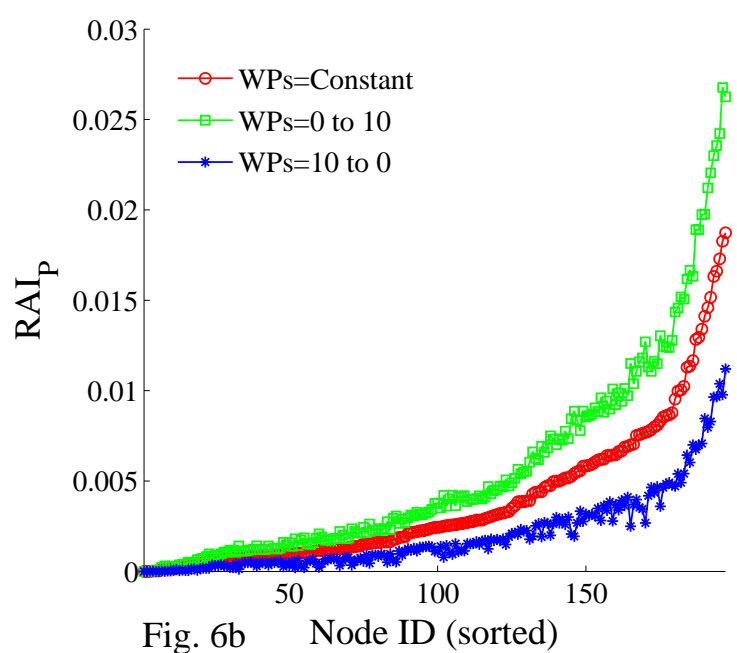

Fig. 6b Node ID (sorted)

Figure 6. $R A I_{P}$ in the European ATN. Fig. $6 \mathrm{~b}$ is using new node IDs based on nodes sorted by their $R A I_{P}$ (with constant weighting parameters).

Both the correlation coefficients and Spearman's rank correlation coefficients among these three sets of $R A I_{P}$ shown in Figure 6 are above 0.97 and the $p$-values are all close to zero, which indicate that our results attain statistical significance. Moreover, the high Spearman's rank correlation coefficients show that the ranking of nodes based on $R A I_{P}$ is insensitive to the change of the weighting parameters.

Figure $6 \mathrm{a}$ also shows that the $R A I_{P}$ of airports (ID $1-67$ ) are generally smaller than the $R A I_{P}$ of aggregated nodes and ACCs (ID 68 - 195). In view of the fact that airport nodes tend to have lower capacities, this result is in line with the anticipation that the influence of nodes with higher capacities is, in general, larger than those with lower capacities, since the capacity reduction is based on percentages.

Table 2 shows that there are five airports and five ACCs with both high $R A I_{P}$ and high capacities, as appeared in the top ten; they are highlighted in bold. However, the $R A I_{P}$ and capacity of some ACCs are counterintuitive. For instance, both the traffic load and capacity of London, Maastricht, Karlsruhe and Marseille are high but their RAIP values are relatively low. In contrast, the $R A I_{P}$ of Bucharest, Bremen, Madrid, Ankara/Istanbul, Belgrade are high while their capacities are comparatively low. These results imply that the importance of a given node in the presence of capacity reduction is not necessarily in line with its capacity.

\begin{tabular}{|c|c|c|c|c|}
\hline \multirow{2}{*}{ Ranking } & \multicolumn{2}{|c|}{ Airport } & \multicolumn{2}{|c|}{ Airspace (ACC) } \\
\hline & $R A I_{P}$ & Capacity & $R A I_{P}$ & Capacity \\
\hline 1 & WIEN SCHWECHAT & PARIS CDG & ROME & LONDON \\
\hline 2 & ISTANBUL ATATURK & $\frac{\text { SCHIPHOL }}{\text { AMSTERDAM }}$ & PRESTWICK & MAASTRICHT \\
\hline 3 & $\begin{array}{l}\text { SCHIPHOL } \\
\text { AMSTERDAM }\end{array}$ & $\underline{\text { KIEV BORISPOL }}$ & $\underline{\text { PARIS }}$ & KARLSRUHE \\
\hline 4 & $\overline{\text { MADRID BARAJAS }}$ & MADRID BARAJAS & LANGEN & MUNICH \\
\hline 5 & MUENCHEN 2 & FRANKFURT & $\overline{\text { BUCHAREST }}$ & $\overline{\text { LONDON TC }}$ \\
\hline 6 & $\begin{array}{l}\text { COPENHAGEN } \\
\text { KASTRUP }\end{array}$ & MUENCHEN 2 & $\underline{\text { MUNICH }}$ & $\underline{\text { PARIS }}$ \\
\hline 7 & OSLO GARDERMOEN & ROME/FIUMICINO & BREMEN & LANGEN \\
\hline 8 & ANTALYA & LONDON HEATHROW & MADRID & ROME \\
\hline 9 & $\underline{\text { KIEV BORISPOL }}$ & STOCKHOLM ARLANDA & $\begin{array}{l}\text { ANKARA/IST } \\
\text { ANBUL }\end{array}$ & MARSEILLE \\
\hline 10 & HELSINKI-VANTAA & $\begin{array}{l}\text { COPENHAGEN } \\
\text { KASTRUP }\end{array}$ & BELGRADE & PRESTWICK \\
\hline
\end{tabular}


The $R A I_{A}$ is calculated by applying capacity reductions to each node by a certain

601

602

603

604

605

606

607

608

609

610

611

612

613

614

615

616

617

618

619

620

621 value (in flight per hour). We set the capacity reductions from 0 to 50 (flight per hour) to examine the influences of absolute-value capacity reductions on network capacity. Similar to the $R A I_{P}$ case, three sets of weighting parameters are considered. The results of the RAIA are shown in Figure 7. A high correlation among the three sets of $R A I_{A}$ is observed, with all the correlation coefficients and Spearman's rank correlation coefficients above 0.99 , and the $p$ values all close to zero. However, in contrast to $R A I_{P}$, no significant difference in the $R A I_{A}$ exists between the airport nodes and the ACC nodes (see Figure 7a). This is partially due to the relatively small capacity reduction (by up to 50 flights per hour) such that most of the airports and ACCs are far from being bottlenecked. Thus the global effects of flow reduction induced by local degradation at airports or ACCs cannot be differentiated.
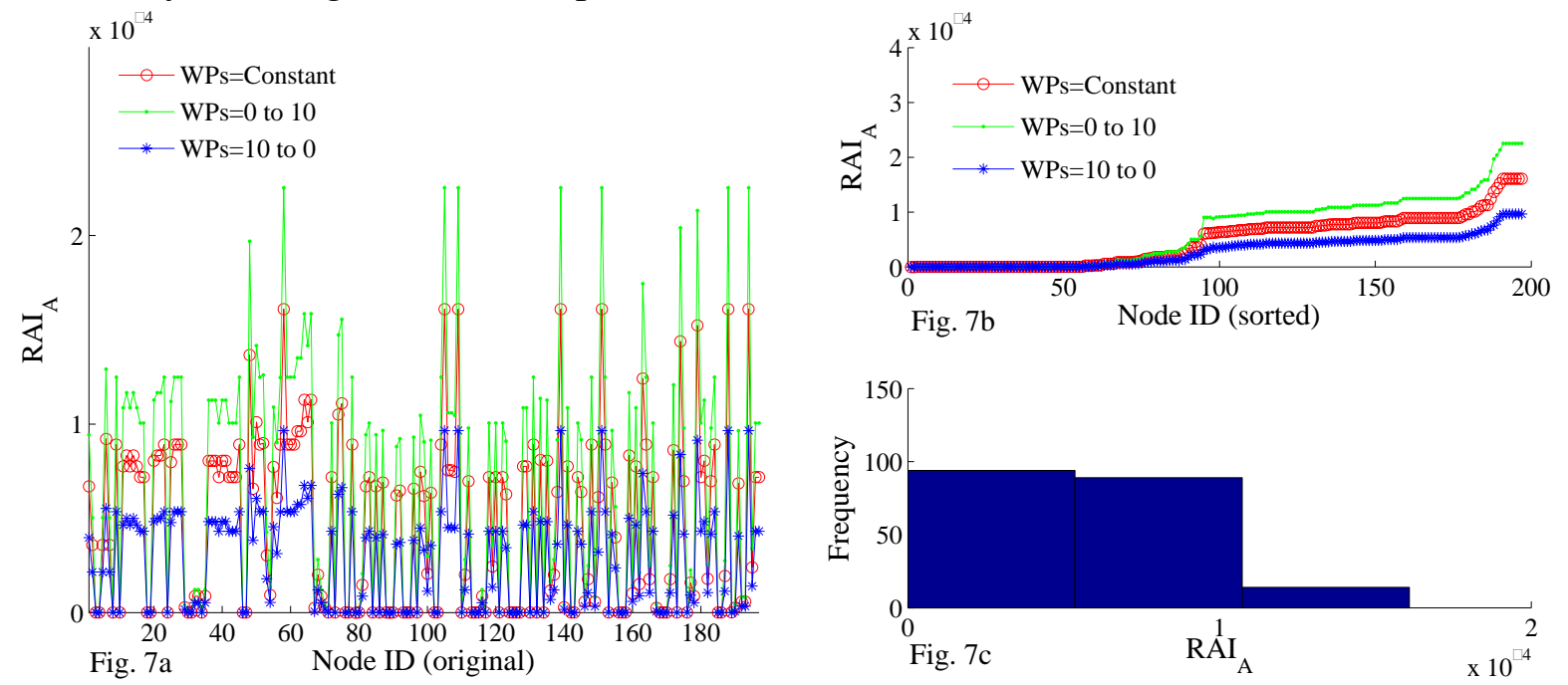

Figure 7. $R A I_{A}$ in the European ATN. Fig. 7b is using new node IDs based on nodes sorted by their $R A I_{A}$ (with constant weighting parameters).

An interesting result of the $R A I_{A}$ is shown when we sort all the nodes in an ascending order with respect to $R A I_{A}$ with constant weighting parameter (Figure $7 \mathrm{~b}$ ): The nodes can be intuitively clustered into three groups according to their $R A I_{A}$, namely low (ID 1 - 95), medium (ID 96 -180), and high (ID 181 - 195); see Figure 7b and Figure 7c.

Table 3 shows that the correlation between $R A I_{A}$ and capacity is comparatively weak, with only a few nodes in common in the top ten. The influence of a minor absolute capacity reduction on the entire network is not in line with the magnitudes of the nodal capacities.

\begin{tabular}{|c|l|l|l|l|}
\hline \multirow{2}{*}{ Ranking } & \multicolumn{2}{|c|}{ Airport } & \multicolumn{2}{c|}{ Airspace (ACC) } \\
\cline { 2 - 5 } & \multicolumn{1}{|c|}{ RAI $_{A}$} & \multicolumn{1}{c|}{ Capacity } & \multicolumn{1}{c|}{ Capacity } \\
\hline 1 & WIEN SCHWECHAT & PARIS CDG & KYIV & LONDON \\
\hline 2 & ATHINAI-E-VENIZELOS & SCHIPHOL AMSTERDAM & PRESTWICK & MAASTRICHT \\
\hline 3 & ISTANBUL ATATURK & KIEV BORISPOL & NICOSIA & KARLSRUHE \\
\hline 4 & ISTANBUL SABIHA & MADRID BARAJAS & BREMEN & MUNICH \\
\hline 5 & MAKEDONIA & FRANKFURT & CANARIAS & LONDON TC \\
\hline 6 & IZMIR ADNAN MENDERES & MUENCHEN 2 & BUCHAREST & PARIS \\
\hline 7 & ANTALYA & ROME/FIUMICINO & $\underline{\text { ROME }}$ & LANGEN \\
\hline 8 & ANKARA ESENBOGA & LONDON HEATHROW & RIGA & ROME \\
\hline
\end{tabular}


622

623

624

625

626

627

628

629

630

631

632

633

634

635

636

637

638

639

640

641

642

643

644

645

646

647

648

649

650

651

652

\begin{tabular}{|c|l|l|l|l|}
\hline 9 & MUENCHEN 2 & STOCKHOLM ARLANDA & PARIS & MARSEILLE \\
\hline 10 & $\begin{array}{l}\text { CATANIA } \\
\text { FONTANAROSSA }\end{array}$ & COPENHAGEN KASTRUP & STOCKHOLM & PRESTWICK \\
\hline
\end{tabular}

Table 3. Top ten airports and airspaces that have the highest $\mathrm{RAI}_{\mathrm{A}}$ and capacities.

\subsection{Comparison between $B C$ and $R A I$}

Given that the focus and the underlying assumptions of $B C$ and $R A I$ are different, it is difficult to conduct a direct comparison between these two robustness indices. Instead, we use relevant empirical data (ATFM delays) to examine the practical relevance of these two indices, and identify their strength and weakness.

Spearman's ranking correlation coefficient is used to reveal the relationships between the ranking of nodes based on the empirical data and the relevant robustness indices. Table 4 shows the correlation coefficients $(r)$ among the six measures: the empirical ATFM delay, capacity, traffic load, $R A I_{P}, R A I_{A}$, and $B C$.

\begin{tabular}{|c|c|r|r|r|r|r|r|}
\hline \multicolumn{2}{|c|}{ Measures } & ATFM delay & Capacity & Traffic load & \multicolumn{1}{c|}{$R A I_{P}$} & \multicolumn{1}{c|}{$R A I_{A}$} & $B C$ \\
\hline \multirow{2}{*}{ ATFM delay } & $r$ & 1.00 & -0.44 & 0.23 & -0.31 & 0.31 & -0.12 \\
\cline { 2 - 8 } & $p$ & 1.00 & 0.00 & 0.00 & 0.00 & 0.00 & 0.10 \\
\hline \multirow{2}{*}{ Capacity } & $r$ & -0.44 & 1.00 & $\mathbf{0 . 4 7}$ & $\mathbf{0 . 7 2}$ & -0.24 & 0.37 \\
\cline { 2 - 8 } & $p$ & 0.00 & 1.00 & 0.00 & 0.00 & 0.00 & 0.00 \\
\hline \multirow{2}{*}{ Traffic load } & $r$ & 0.23 & $\mathbf{0 . 4 7}$ & 1.00 & $\mathbf{0 . 5 1}$ & 0.19 & $\mathbf{0 . 5 1}$ \\
\cline { 2 - 8 } & $p$ & 0.00 & 0.00 & 1.00 & 0.00 & 0.01 & 0.00 \\
\hline \multirow{2}{*}{$R A I_{P}$} & $r$ & -0.31 & $\mathbf{0 . 7 2}$ & $\mathbf{0 . 5 1}$ & 1.00 & 0.31 & 0.37 \\
\cline { 2 - 8 } & $p$ & 0.00 & 0.00 & 0.00 & 1.00 & 0.00 & 0.00 \\
\hline \multirow{2}{*}{$R A I_{A}$} & $r$ & 0.31 & -0.24 & 0.19 & 0.31 & 1.00 & 0.13 \\
\cline { 2 - 8 } & $p$ & 0.00 & 0.00 & 0.01 & 0.00 & 1.00 & 0.06 \\
\hline \multirow{2}{*}{$B C$} & $r$ & -0.12 & 0.37 & 0.51 & 0.37 & 0.13 & 1.00 \\
\cline { 2 - 8 } & $p$ & 0.10 & 0.00 & 0.00 & 0.00 & 0.06 & 1.00 \\
\hline
\end{tabular}

Table 4. Spearman's ranking correlation coefficients and the $p$-values among relevant indices and empirical data (ATFM delay)

Spearman's ranking correlation coefficient $r$ between $R A I_{P}$ and the traffic load is comparable with that between $B C$ and the traffic load (0.51). Secondly, the ranking of the $R A I_{P}$ is strongly correlated to the ranking of the nodal capacity (0.72), showing $R A I_{P}$ to be an index more promising than $B C$ to capture the nodal capacity. Thirdly, $R A I_{A}$ has no meaningful correlation with any of the other indices. This indicates that the influence of a minor, absolute capacity reduction of a given node is not significantly related to traffic load, nodal capacity, or ATFM delays. However, we expect that when the absolute capacity reduction gets larger, say for a subset of the nodes that have large capacities, $\mathrm{RAI}_{\mathrm{A}}$ is likely to provide a more meaningful characterization of the importance of nodes.

In Table 4, neither the $R A I$ nor the $B C$ captures the ATFM delays. This is explained by the fact that the ATFM delay is a result of a complex operational environment, involving multiple sectors and stakeholders; thus more sophisticated models are required to capture the ATFM delays in their entirety.

In order to further compare $R A I$ and $B C$, we select the top 25 nodes with the highest ATFM delays ${ }^{1}$, which are subsequently referred to as bottlenecks, and conduct a similar analysis restricted to these 25 bottlenecks. The findings are presented in Table 5. Here, BC again provides a poor performance with low Spearman's ranking correlation coefficient and high $p$-values. An important finding is that Spearman's ranking correlation coefficient

${ }^{1}$ These 25 nodes include 21 airports and 4 ACCs, with ATFM delays greater than the mean (3.9 minutes); see the Appendix for more information. 
653

654

655

656

657

658

659

660

661

662

663

664

665

666

667

668

669

670

671

672

673

674

675

676

677

678

679

680

681

682

683

684

685

686

687

688

689

between $R A I_{A}$ and $R A I_{P}$ increases from 0.31 to 0.72 , which means that the capacity reductions by percentage or absolute value have similar effects for these 25 nodes. In addition, both $R A I_{A}$ and $R A I_{P}$ better capture the ATFM delays at these bottlenecks, with $r=$ 0.44 and $r=0.40$, respectively.

\begin{tabular}{|c|c|r|r|r|r|r|r|}
\hline \multicolumn{2}{|c|}{ Measures } & ATFM delay & \multicolumn{1}{|c|}{ Capacity } & Traffic load & \multicolumn{1}{c|}{$R A I_{P}$} & \multicolumn{1}{c|}{$R A I_{A}$} & \multicolumn{1}{c|}{$B C$} \\
\hline \multirow{2}{*}{ ATFM delay } & $r$ & 1.00 & 0.06 & 0.23 & $\mathbf{0 . 4 4}$ & $\mathbf{0 . 4 0}$ & 0.19 \\
\cline { 2 - 8 } & $p$ & 1.00 & 0.78 & 0.28 & $\mathbf{0 . 0 3}$ & $\mathbf{0 . 0 5}$ & 0.36 \\
\hline \multirow{2}{*}{ Capacity } & $r$ & 0.06 & 1.00 & 0.82 & $\mathbf{0 . 4 8}$ & -0.03 & 0.22 \\
\cline { 2 - 8 } & $p$ & 0.78 & 1.00 & 0.00 & $\mathbf{0 . 0 2}$ & 0.90 & 0.28 \\
\hline \multirow{2}{*}{ Traffic load } & $r$ & 0.23 & 0.82 & 1.00 & $\mathbf{0 . 6 1}$ & 0.16 & 0.30 \\
\cline { 2 - 8 } & $p$ & 0.28 & 0.00 & 1.00 & $\mathbf{0 . 0 0}$ & 0.45 & 0.15 \\
\hline \multirow{2}{*}{$R A I_{P}$} & $r$ & $\mathbf{0 . 4 4}$ & $\mathbf{0 . 4 8}$ & $\mathbf{0 . 6 1}$ & 1.00 & $\mathbf{0 . 7 2}$ & 0.38 \\
\cline { 2 - 8 } & $p$ & $\mathbf{0 . 0 3}$ & $\mathbf{0 . 0 2}$ & $\mathbf{0 . 0 0}$ & 1.00 & $\mathbf{0 . 0 0}$ & 0.06 \\
\hline \multirow{2}{*}{$R A I_{A}$} & $r$ & $\mathbf{0 . 4 0}$ & -0.03 & 0.16 & $\mathbf{0 . 7 2}$ & 1.00 & 0.23 \\
\cline { 2 - 8 } & $p$ & $\mathbf{0 . 0 5}$ & 0.90 & 0.45 & $\mathbf{0 . 0 0}$ & 1.00 & 0.27 \\
\hline \multirow{2}{*}{$B C$} & $r$ & 0.19 & 0.22 & 0.30 & 0.38 & 0.23 & 1.00 \\
\cline { 2 - 8 } & $p$ & 0.36 & 0.28 & 0.15 & 0.06 & 0.27 & 1.00 \\
\hline
\end{tabular}

Table 5. Spearman's ranking correlation coefficients and the $p$-values among relevant indices and empirical data (ATFM delay), among the 25 high-ATFM-delay nodes.

\section{Discussion}

The commonly employed network robustness index, namely betweenness centrality $(B C)$, reflects only the topological characteristics of the network, without taking into account traffic demand and nodal capacities. As a result, it can only capture traffic loads rather than capacity or ATFM delays, as we show in our results for the European air traffic network. Using $B C$ as the robustness index of an ATN is quite limited in capturing the influence of local disruption on the network level, especially when the operational characteristics are within the purview of network operators.

Our finding that the $B C$ is capable of capturing the actual traffic load at a particular node differs from that of Cats and Jenelius (2014), who found limited correlation between the passenger loads and $B C s$ in a road network. We argue that this difference is caused by the different nature of road and air traffic. Compared to road users who are free to minimize their cost of travel by selecting alternative routes in a networks, aircrafts do not have the freedom to select alternative routes; instead, they fly along the routes in given flight plans and follow the guidance of air traffic control (ATC). In addition, the delay of air traffic often occurs at the departing airport as a result of air traffic management, while congestion and delays of road traffic take place en route. These differences imply that the $B C$ may be an adequate indicator for air traffic volume in an ATN, since the shortest distance is an important factor in the design of flight plans.

The proposed robustness index $(R A I)$ is more capable than the $B C$ of capturing the importance of a given node in the event of capacity reduction, by considering traffic demands, actual flight paths, and nodal capacities, in addition to the topological features. It also encapsulates a range of scenarios involving different levels of capacity reductions, instead of simply removing a node or a link, which is typical in topological analysis leading up to $\mathrm{BC}$ and other indices. The concept and formulation of $R A I$ is flexible enough to accommodate a wide range of cases involving different nature and severity of the capacity reduction. We first adopt the percentage-based capacity reduction in combination with a network flow maximisation technique for assessing the theoretical network capacity. We find that the $R A I_{P}$ ranking at the aggregated nodes and ACCs are generally higher than those at the airport nodes. Although the use of three sets of weighting parameters results in different 
rankings of nodes, the difference is extremely small. However, we note that the importance of the weighting parameters should be re-evaluated by introducing more sophisticated modelling elements, such as dynamic network modelling and routing and scheduling.

In addition to the percentage-based capacity reductions, we also applied the absolute capacity reduction to calculate $R A I_{A}$. This approach enables us to assess the influence of a certain event that causes absolute capacity reductions at given nodes. Unlike the $R A I_{P}$ ranking, the result shows that the $R A I_{A}$ ranking do not favour the high-capacity nodes such as the ACCs and aggregated nodes. In addition to the ranking, the $R A I_{A}$ of the nodes in the European ATN can be intuitively categorized into three groups: High-, Medium- and Low$R A I_{A}$ nodes. A particular event such as sector- or runway-closure at the nodes in the High$R A I_{A}$ group will cause a greater impact on network capacity than if that event happens at the nodes in the Medium- and Low- $R A I_{A}$ groups. This functionality provides the network management unit with a powerful tool to group and rank the critical nodes, not solely using the empirical delay data that may contain considerable inaccuracies. Compared to the $\mathrm{BC}$, using the $R A I$ is more flexible to assess network robustness (see $R A I_{P}$ in Table 4 and $R A I_{A}$ in Figure 7). Therefore, the proposed new index has the potential to be used to reflect the ranking of the constituent nodes in an ATN and to assess network robustness. Table 5 shows the superiority of $R A I$ over $B C$ at the 25 main bottlenecks.

There are potentially four extensions of this paper for future research. First, the formulation of the conventional index, $B C$ can be improved to accommodate the features of air traffic, including traffic demands, flight routes and the heterogeneity between the airport and airspace nodes. Second, since the European ATN is not saturated and the capacity of each node varies dynamically, there is a need to capture network flows dynamically by introducing dynamic capacity constraints and flight times. Third, both the $R A I_{A}$ and $R A I_{P}$ require real data on the influence of capacity fluctuation on network capacity for their validation. This validation would enable the superiority of the RAI when compared to the current KPI of ATFM delays to be evaluated. Finally, unexpected events that occur in realtime, such as large-scale meteorological events and industrial action, may reduce the capacity at multiple airports and en-route airspace. Hence, any future analysis would benefit from an evaluation of the influence of capacity-reductions at multiple nodes in the network simultaneously, rather than at just a single node.

\section{Conclusion}

This paper proposes a new index, the $R A I$, to assess the robustness of the European ATN by calculating the influence of nodal capacity-reductions on network capacity. Using data from three of the busiest air traffic days in Europe in 2012, the $R A I$ was assessed along topological index, the $B C$, as potential indicators for robustness. The results indicate that the $R A I$ is better able to capture the importance of each node by taking into account not only the topological features, but also the traffic demands and the nodal capacities.

There are several potential operational applications of the RAI to air traffic management. Compared to ATFM delay that is the current indicator of network capacity and of any bottlenecks in the network, the RAI provides a detailed ranking of the nodes in the European ATN from the standpoint of any potential local degradation of capacity and its consequent impact on the overall network. Such a consideration has not been addressed by the ATFM delay, the $B C$ or any other existing network performance indices for air traffic networks. RAI therefore provides a potentially powerful tool for the European ATM unit to identify and categorize the critical nodes in the network. This in turn can aid in improving network management and resource allocations, by identifying nodes with higher 'marginal benefits'. 
Given the expected rise in air traffic demand in Europe in the coming years, SESAR is effectively revolutionising the nature of air traffic operations and their management in

740 Europe. However, as EUROCONTROL noted in their "Challenges for growth 2013"

741 (EUROCONTROL, 2013a), there is an urgent need to understand network performance in the

742 future European air traffic network and then to have appropriate metrics for this performance,

743 to a far greater degree of sophistication than the current ATFM delays. Given this need, we

744 recommend the use of the $R A I$ methodology for the development of the future of European

745 network performance indicators.

\section{Acknowledgement}

747 The authors thank the EUROCONTROL for providing the data of flight profiles and 748 ATFM delays. We also thank Dr. Xiaoqian Sun who generously provided constructive 749 suggestions on network robustness. 


\section{References}

Barrat, A., M. Barthelemy, R. Pastor-Satorras, \& A. Vespignani. 2004. "The architecture of complex weighted networks". Proceedings of the National Academy of Sciences of the United States of America, 101(11), 3747-3752.

Brandes, U. 2001. "A faster algorithm for betweenness centrality*". Journal of Mathematical Sociology, 25(2), 163-177.

Cats, O., \& E. Jenelius. 2014. "Dynamic vulnerability analysis of public transport networks: mitigation effects of real-time information". Networks and Spatial Economics, 14(3-4), 435-463.

Dehmer, M. (2011). Structural Analysis of Complex Networks: Springer.

Di Paolo, E., K. Zhang, S. Yang, X.-B. Hu, \& H. Liu. 2011. "Application of Complex Network Theory and Genetic Algorithm in Airline Route Networks". Transportation Research Record: Journal of the Transportation Research Board, 2214(-1), 50-58.

EUROCONTROL. (2007). Capacity Assessment \& Planning Guidance-An overview of the European Network Capacity Planning Process. Brussels, Belgium.

EUROCONTROL. (2013a). Challenges of Growth 2013. Brussels, Belgium.

EUROCONTROL. (2013b). European Network Operations Plan 2013-2015. Brussels, Belgium.

EUROCONTROL. 2013c, "European Route Network Improvement Plan Part 2-European ATS Route Network - Version 2013-2015", Brussels, Belgium.

EUROCONTROL. (2014). Network Operations Report 2013. Brussels, Belgium.

EUROCONTROL. (2015). Seven-Year Flight Movements and Service Units Forecast: 20152021. Brussels, Belgium.

EUROCONTROL/FAA. (2009). U.S./Europe Comparison of ATM-related Operational Performance.

Freeman, L. C. 1979. "Centrality in social networks conceptual clarification". Social networks, 1(3), 215-239.

Geraci, A., F. Katki, L. McMonegal, B. Meyer, J. Lane, P. Wilson, ... F. Springsteel. (1991). IEEE standard computer dictionary: Compilation of IEEE standard computer glossaries: IEEE Press.

Guimera, R., \& L. A. N. Amaral. 2004. "Modeling the world-wide airport network". The European Physical Journal B-Condensed Matter and Complex Systems, 38(2), 381385.

Holme, P., B. J. Kim, C. N. Yoon, \& S. K. Han. 2002. "Attack vulnerability of complex networks". Physical Review E, 65(5), 056109.

Holmes, B. J., Scott, John M. (2004). Transportation network topologies. Paper presented at the Integrated Communications, Navigation, and Surveillance (ICNS) Conference, Fairfax, Virginia, USA.

Holmgren, Å. J. (2007). A framework for vulnerability assessment of electric power systems Critical Infrastructure (pp. 31-55): Springer.

Immers, B., I. Yperman, J. Stada, \& A. Bleukx. 2004. "Reliability and robustness of transportation networks: problem survey and examples". Proceedings of the NECTAR cluster meeting on reliability of networks, Amsterdam, the Netherlands, 19-20.

Jahn, J. (2007). Introduction to the theory of nonlinear optimization: Springer.

Lordan, 0., J. M. Sallan, P. Simo, \& D. Gonzalez-Prieto. 2014. "Robustness of the air transport network". Transportation Research Part E: Logistics and Transportation Review, 68(0), 155-163. 
Nagurney, A., \& Q. Qiang. 2007a. "A network efficiency measure for congested networks". EPL (Europhysics Letters), 79(3), 38005.

Nagurney, A., \& Q. Qiang. 2007b. "Robustness of transportation networks subject to degradable links". EPL (Europhysics Letters), 80(6), 68001.

Nagurney, A., \& Q. Qiang. 2009. "A relative total cost index for the evaluation of transportation network robustness in the presence of degradable links and alternative travel behavior". International Trabsactions in Operation Research, 16, 49-67.

Newman, M. E. 2001. "Scientific collaboration networks. II. Shortest paths, weighted networks, and centrality". Physical Review E, 64(1), 016132.

Pien, K.-C., A. Majumdar, K. Han, \& W. Y. Ochieng. 2014. "A linear programming approach to maximum flow estimation on the European air traffic network ". in the 6th International Conference on Research in Air Transportation (ICRAT2014), 2014: Istanbu Technical University, Turkey.

Sakakibara, H., Y. Kajitani, \& N. Okada. 2004. "Road Network Robustness for Avoiding Functional Isolation in Disasters". Journal of Transportation Engineering, 130(5), 560-567.

Scott, D. M., D. Novak, L. Aultman-Hall, \& F. Guo. 2005. "Network robustness index- a new method for identifying critical links and evaluating the perfomance of transportation networks ". Center for spatial analysis working paper.

Sun, X., \& S. Wandelt. 2014. "Network similarity analysis of air navigation route systems". Transportation Research Part E: Logistics and Transportation Review, 70, 416-434.

Sun, X., S. Wandelt, \& F. Linke. 2014. "Topological Properties of the Air Navigation Route System using Complex Network Theory". in the 6th International Conference on Research in Air Transportation (ICRAT2014), 2014:Istanbu Technical University, Turkey.

Wasserman, S. (1994). Social network analysis: Methods and applications (Vol. 8): Cambridge university press.

Wei, P., L. Chen, \& D. Sun. 2014. "Algebraic connectivity maximization of an air transportation network: The flight routes' addition/deletion problem". Transportation Research Part E: Logistics and Transportation Review, 61(0), 1327.

Zhao, C., B. Fu, \& T. Wang. 2014. "Braess paradox and robustness of traffic networks under stochastic user equilibrium". Transportation Research Part E: Logistics and Transportation Review, 61(0), 135-141. 


\section{Appendix}

The table below is concerned with the top 25 nodes in the European Air Traffic Network in terms of ATFM delays. Other performance indices of interest, including capacity, traffic load, $R A I$ and $B C$ are computed and presented for these 25 nodes, along with their rankings in each of the categories.

\begin{tabular}{|c|c|c|c|c|c|c|c|c|c|c|c|c|c|c|c|}
\hline & \multirow{2}{*}{ Nodes } & \multirow{2}{*}{ Type } & \multirow{2}{*}{ ID } & \multicolumn{2}{|c|}{ ATFM delays } & \multicolumn{2}{|c|}{ Capacity } & \multicolumn{2}{|c|}{ Traffic } & \multicolumn{4}{|c|}{ RAI } & \multicolumn{2}{|c|}{ Betweenness } \\
\hline & & & & $\min / f l t$ & ranking & flt/day & ranking & flt/day & ranking & $R A I_{P}$ & ranking & $R A I_{A}$ & ranking & $\mathrm{BC}$ & ranking \\
\hline 1 & BARCELONA & Airport & LEBL & 18.74 & 1 & 1056 & 9 & 926 & 6 & 0.00191 & 6 & 0.00008 & 8 & 30 & 9 \\
\hline 2 & MUENCHEN 2 & Airport & EDDM & 12.32 & 2 & 1440 & 4 & 1060 & 4 & 0.00283 & 3 & 0.000092 & 2 & 0 & 11 \\
\hline 3 & \begin{tabular}{|l} 
PALMA-DE- \\
MALLORCA
\end{tabular} & Airport & LEPA & 11.78 & 3 & 992 & 10 & 836 & 7 & 0.00162 & 8 & 0.00008 & 9 & 0 & 11 \\
\hline 4 & $\begin{array}{l}\text { WARSZAWA } \\
\text { OKECIE }\end{array}$ & Airport & EPWA & 8.74 & 4 & 640 & 18 & 397 & 17 & 0.00016 & 24 & 0.000003 & 21 & 0 & 11 \\
\hline 5 & ALICANTE & Airport & LEAL & 8.48 & 5 & 480 & 22 & 228 & 24 & 0.00088 & 14 & 0.00008 & 10 & 0 & 11 \\
\hline 6 & VALENCIA & Airport & LEVC & 8.24 & 6 & 480 & 23 & 174 & 25 & 0.00088 & 15 & 0.00008 & 11 & 2226 & 3 \\
\hline 7 & BARCELONA & $\mathrm{ACC}$ & LECBCTA & 7.78 & 7 & 3288 & 2 & 2893 & 2 & 0.00264 & 4 & 0.000009 & 19 & 397 & 5 \\
\hline 8 & NICOSIA & ACC & LCCCCTA & 7.66 & 8 & 1200 & 6 & 976 & 5 & 0.00386 & 2 & 0.000161 & 1 & 380 & 6 \\
\hline 9 & ZURICH & Airport & LSZH & 6.51 & 9 & 1152 & 7 & 788 & 9 & 0.00211 & 5 & 0.000089 & 3 & 0 & 11 \\
\hline 10 & \begin{tabular}{|l} 
GENEVE \\
COINTRIN \\
\end{tabular} & Airport & LSGG & 6.35 & 10 & 640 & 19 & 524 & 13 & 0.00126 & 12 & 0.000089 & 4 & 1439 & 4 \\
\hline 11 & NICE & Airport & LFMN & 5.52 & 11 & 832 & 12 & 625 & 10 & 0.00159 & 9 & 0.000089 & 5 & 222 & 7 \\
\hline 12 & HERAKLION & Airport & LGIR & 5.52 & 12 & 352 & 25 & 261 & 21 & 0.0006 & 21 & 0.000066 & 17 & 0 & 11 \\
\hline 13 & DUESSELDORF & Airport & EDDL & 5.39 & 13 & 720 & 17 & 587 & 11 & 0.00061 & 20 & 0.000036 & 18 & 0 & 11 \\
\hline 14 & PALMA & $\mathrm{ACC}$ & LECPCTA & 5.17 & 14 & 2208 & 3 & 1356 & 3 & 0.00646 & 1 & 0.00008 & 12 & 3575 & 1 \\
\hline 15 & BIRMINGHAM & Airport & EGBB & 4.88 & 15 & 640 & 20 & 248 & 23 & 0.00101 & 13 & 0.000078 & 14 & 215 & 8 \\
\hline 16 & \begin{tabular}{|l} 
LONDON \\
STANSTED \\
\end{tabular} & Airport & EGSS & 4.68 & 16 & 800 & 14 & 411 & 16 & 0.00132 & 11 & 0.00008 & 13 & 0 & 11 \\
\hline 17 & MALAGA & Airport & LEMG & 4.59 & 17 & 560 & 21 & 416 & 15 & 0.00088 & 16 & 0.000072 & 15 & 0 & 11 \\
\hline 18 & KARLSRUHE & $\mathrm{ACC}$ & EDMMCTA & 4.5 & 18 & 7176 & 1 & 4301 & 1 & 0.00076 & 17 & 0 & 22 & 2782 & 2 \\
\hline 19 & \begin{tabular}{|l} 
PRAHA \\
RUZYNE
\end{tabular} & Airport & LKPR & 4.41 & 19 & 736 & 16 & 376 & 18 & 0.00163 & 7 & 0.000089 & 6 & 0 & 11 \\
\hline 20 & KOELN-BONN & Airport & EDDK & 4.3 & 20 & 1280 & 5 & 288 & 20 & 0.0002 & 23 & 0 & 22 & 0 & 11 \\
\hline 21 & \begin{tabular}{|l} 
MILANO \\
MALPENSA \\
\end{tabular} & Airport & LIMC & 4.27 & 21 & 1120 & 8 & 562 & 12 & 0.00068 & 19 & 0.000009 & 20 & 0 & 11 \\
\hline 22 & HAMBURG & Airport & EDDH & 4.18 & 22 & 768 & 15 & 372 & 19 & 0.00013 & 25 & 0 & 22 & 0 & 11 \\
\hline 23 & $\begin{array}{l}\text { FERIHEGY } \\
\text { BUDAPEST }\end{array}$ & Airport & LHBP & 4.17 & 23 & 384 & 24 & 259 & 22 & 0.00074 & 18 & 0.000089 & 7 & 15 & 10 \\
\hline 24 & $\begin{array}{l}\text { LONDON } \\
\text { GATWICK } \\
\end{array}$ & Airport & EGKK & 4.06 & 24 & 960 & 11 & 797 & 8 & 0.00152 & 10 & 0.000072 & 16 & 0 & 11 \\
\hline 25 & $\begin{array}{l}\text { TEGEL- } \\
\text { BERLIN }\end{array}$ & Airport & EDDT & 4.04 & 25 & 832 & 13 & 427 & 14 & 0.00023 & 22 & 0 & 22 & 0 & 11 \\
\hline
\end{tabular}

